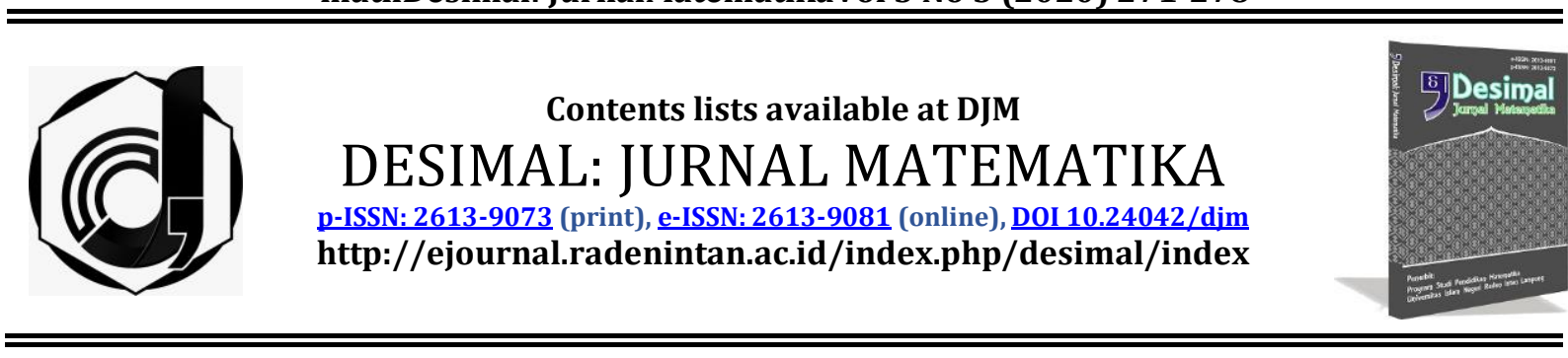

\title{
The Numerical Simulation for Asymptotic Normality of the Intensity Obtained as a Product of a Periodic Function with the Power Trend Function of a Nonhomogeneous Poisson Process
}

\author{
Ikhsan Maulidi ${ }^{1,}$, Mahyus Ihsan $^{1}$, Vina Apriliani ${ }^{2}$ \\ ${ }^{1}$ Syiah Kuala University, Banda Aceh, Indonesia \\ 2 UIN Ar-Raniry, Banda Aceh, Indonesia
}

\author{
ARTICLE INFO

\section{Article History} \\ Received :09-05-2020 \\ Revised :08-09-2020 \\ Accepted : 28-09-2020 \\ Published : 30-09-2020

\section{Keywords:} \\ Poisson Process, Intensity \\ Function, Power Trend Function, \\ Asymptotic Normality. \\ *Correspondence: E-mail: \\ ikhsanmaulidi@unsyiah.ac.id \\ Doi: \\ $\underline{10.24042 / \text { djm.v3i3.6374 }}$
}

\begin{abstract}
In this article, we provided a numerical simulation for asymptotic normality of a kernel type estimator for the intensity obtained as a product of a periodic function with the power trend function of a nonhomogeneous Poisson Process. The aim of this simulation is to observe how convergence the variance and bias of the estimator. The simulation shows that the larger the value of power function in intensity function, it is required the length of the observation interval to obtain the convergent of the estimator.
\end{abstract}

\section{INTRODUCTION}

There are many real phenomena that can be modeled using stochastic processes. This kind of model uses opportunity rules that describe the behavior of a system that is not known in the future. For example, the process of coming customers to a service center such as bank, post office, supermarket, etc ((Mahmud et al., n.d.; Sumiati et al., 2019; Tse, 2014)). The Poisson process is one of the continuous stochastic processes, namely the enumeration process where the number of events at time intervals spreads with the Poisson distribution.

The Poisson process is divided into two, namely the homogeneous Poisson process and the nonhomogeneous Poisson process. In a homogeneous Poisson process, the intensity function (a function of expectation value) is a constant function (not dependent on time), whereas the intensity function in the nonhomogeneous Poisson process depends on time. Periodic 
Poisson process is a nonhomogeneous Poisson process whose intensity function is a periodic function. This process is one of the special forms of stochastic processes with a continuous time that have many benefits to modeling various kinds of real phenomena related to opportunity rules. Some applications of this periodic Poisson process can be applied in the fields of communication, hydrology, meteorology, insurance, and seismology, (Sumiati et al., 2019),

Suppose $\mathrm{N}$ is a nonhomogeneous Poisson process at interval $[0, n](n$ is an integer number) with an unknown function of intensity $\lambda$ and assumed that the intensity function is locally integrated. It is also assumed that the function of this intensity is the product of the periodic component and the power trend component $a s^{b}$ with $\mathrm{b}>0$ is known, so that for all $s \in[0, \infty]$ the function of intensity $\lambda$ (s) can be expressed as

$$
\lambda(s)=\left(\lambda_{c}^{*}(s)\right) a s^{b}(1)
$$

with $\lambda_{c}^{*}(s)$ is a periodic function with a known period. The constant $a$ is the slope of the trend with $a>0$. Equation (1) can also be written as

$$
\lambda(s)=\left(a \lambda_{c}^{*}(s)\right) s^{b},
$$

with $a \lambda_{c}^{*}(s)$ is also a periodic function. Suppose $\lambda_{c}(s)=a \lambda_{c}^{*}(s)$, then Equation (2) becomes

$$
\lambda(s)=\left(\lambda_{c}(s)\right) s^{b} .
$$

Furthermore, because $\lambda_{c}(s)$ is a periodic function, then $\lambda_{c}(s)=\lambda_{c}(s+k \tau)$, where $\tau$ is the period and $k$ is the natural number. Let $K: \mathbb{R} \rightarrow \mathbb{R}$ is a real value function. The $K$ function is called the kernel if it fulfills the properties: (K1) $K$ is an opportunity density function, (K2) $K$ is bounded, and (K3) $K$ has a domain in $[-1,1]$,(Helmers et al., 2003).

The proposed estimator $\lambda_{c}(s)$ at $s \in$ $[0, \tau]$ has been studied in (Erliana et al., 2014) by only using a single realization $N(\omega)$ from a Poisson process with the intensity function $\lambda(s)$ as in Equation (3) observed in interval $[0, n]$. The estimator is as follows:

$$
\begin{aligned}
& \hat{\lambda}_{c, n, K}(s) \\
& =\frac{\tau}{n} \sum_{k=0}^{\infty} \frac{1}{h_{n}(s+k \tau)^{b}} \int_{0}^{n} K\left(\frac{x-(s+k \tau)}{h_{n}}\right) N(d x) .
\end{aligned}
$$

In the previous article, (Erliana et al., 2014), also has been given the bias, variance, and optimal bandwidth for all values b. Strong consistency of the estimator has also been proven in(Maulidi et al., 2015). Other similar studies can be seen in (Mangku, 2011), (Erliana et al., 2014) and (Mangku et al., 2013).

\section{METHOD}

This research started from the Theorems that analitically prove the normal distribution of the estimator for all cases of $\boldsymbol{b}$ values. The normality asymptotic of the estimator is a useful tool in developing a prediction model. This is because by knowing the asymptotic normality, the interval confident of the estimator can be obtained. Here we provide the theorems for the asymptotic normality of the estimator that has been formulated, Equation (4).

\section{Theorem 1 (Asymptotic Normality of $\widehat{\lambda}_{\boldsymbol{c}, \boldsymbol{n}, \boldsymbol{K}}(\boldsymbol{s})$ for $\mathbf{0}<b<1$ )}

Suppose that the intensity $\lambda$ satisfies (1) and locally integrable. The kernel function $K$ satisfies (K1), (K2), (K3), $\lambda_{c}$ has a bounded second derivative around of $s, 0<b<1, n^{1-b} h_{n} \rightarrow 0, n^{b+1} h_{n} \rightarrow$ $\infty, h_{n} \downarrow 0$ as $n \rightarrow \infty$,

$$
\begin{aligned}
& \text { a) If }\left(n^{1+b} h_{n}^{5}\right)^{\frac{1}{2}} \rightarrow 0 \text {, then } \\
& \left(n^{1+b} h_{n}\right)^{\frac{1}{2}}\left(\hat{\lambda}_{c, n, K}(s)-\lambda_{c}(s)\right) \\
& \stackrel{d}{\rightarrow} \operatorname{Normal}\left(0, \sigma^{2}\right)
\end{aligned}
$$

as $n \rightarrow \infty$, with $\sigma^{2}=\frac{\tau \lambda_{c}(s)}{(1-b)} \int_{-1}^{1} K^{2}(z) d z$.

$$
\begin{aligned}
& \text { b) If }\left(n^{1+b} h_{n}^{5}\right)^{\frac{1}{2}} \rightarrow 1 \text {, then } \\
& \quad\left(n^{1+b} h_{n}\right)^{\frac{1}{2}}\left(\hat{\lambda}_{c, n, K}(s)-\lambda_{c}(s)\right) \\
& \stackrel{d}{\rightarrow} \operatorname{Normal}\left(\mu, \sigma^{2}\right)
\end{aligned}
$$

as $n \rightarrow \infty$, with $\mu=\frac{\lambda_{c}^{\prime \prime}(s)}{2} \int_{-1}^{1} z^{2} K(z) d z$, 
and $\sigma^{2}=\frac{\tau \lambda_{c}(s)}{(1-b)} \int_{-1}^{1} K^{2}(z) d z$.

\section{Theorem 2 (Asymptotic Normality of} $\hat{\lambda}_{c, n, K}(s)$ for $\left.b=1\right)$

Suppose that the intensity $\lambda$ satisfies (1) and locally integrable. The kernel function $K$ satisfies (K1), (K2), (K3), $\lambda_{c}$ has a bounded second derivative around of $s, b=1, \ln (n) h_{n} \rightarrow 0, \frac{n^{2} h_{n}}{\ln (n)} \rightarrow \infty, h_{n} \downarrow 0$ as $n \rightarrow \infty$,

a) If $\left(\frac{n^{2} h_{n}^{5}}{\ln (n)}\right)^{\frac{1}{2}} \rightarrow 0$, then

$$
\begin{array}{r}
\left(\frac{n^{2} h_{n}}{\ln (n)}\right)^{\frac{1}{2}}\left(\hat{\lambda}_{c, n, K}(s)-\lambda_{c}(s)\right) \\
\stackrel{d}{\rightarrow} \operatorname{Normal}\left(0, \sigma^{2}\right)
\end{array}
$$

as $n \rightarrow \infty$, with $\sigma^{2}=\tau \lambda_{c}(s) \int_{-1}^{1} K^{2}(z) d z$.

b) If $\left(\frac{n^{2} h_{n}^{5}}{\ln (n)}\right)^{\frac{1}{2}} \rightarrow 1$, then

$$
\begin{array}{r}
\left(\frac{n^{2} h_{n}}{\ln (n)}\right)^{\frac{1}{2}}\left(\hat{\lambda}_{c, n, K}(s)-\lambda_{c}(s)\right) \\
\stackrel{d}{\rightarrow} \operatorname{Normal}\left(\mu, \sigma^{2}\right)
\end{array}
$$

as $n \rightarrow \infty$, with $\mu=\frac{\lambda_{c}^{\prime \prime}(s)}{2} \int_{-1}^{1} z^{2} K(z) d z$,

and $\sigma^{2}=\tau \lambda_{c}(s) \int_{-1}^{1} K^{2}(z) d z$.

\section{Theorem 3 (Asymtotic Normality of $\hat{\lambda}_{c, n, K}(s)$ for $\boldsymbol{b}>1$ )}

Suppose that the intensity $\lambda$ satisfies (1) and locally integrable. The kernel function $K$ satisfies (K1), (K2), (K3), and $\lambda_{c}$ has a bounded second derivative around of $s, b>1, n^{2} h_{n} \rightarrow \infty, h_{n} \downarrow 0$ as $n \rightarrow \infty$,

a) If $\left(n^{2} h_{n}^{5}\right)^{\frac{1}{2}} \rightarrow 0$, then

$$
\begin{aligned}
\left(n^{2} h_{n}\right)^{\frac{1}{2}}\left(\hat{\lambda}_{c, n, K}(s)-\lambda_{c}(s)\right) & \stackrel{d}{\rightarrow} \operatorname{Normal}\left(0, \sigma^{2}\right)
\end{aligned}
$$

Asn $\rightarrow \infty$ with $\quad \sigma^{2}=$ $\tau^{2-b} \lambda_{c}(s) \zeta(b) \int_{-1}^{1} K^{2}(z) d z$ and $\quad \zeta(b)=$ $\lim _{n \rightarrow \infty}\left(\sum_{k=1}^{\infty} \frac{1}{k^{b}} I(x+s+k \tau \in[0, n])\right)$.

b) If $\left(n^{2} h_{n}^{5}\right)^{\frac{1}{2}} \rightarrow 1$, then

$$
\begin{aligned}
&\left(n^{2} h_{n}\right)^{\frac{1}{2}}\left(\hat{\lambda}_{c, n, K}(s)-\lambda_{c}(s)\right) \\
& \stackrel{d}{\rightarrow} \operatorname{Normal}\left(\mu, \sigma^{2}\right)
\end{aligned}
$$

as $\quad n \rightarrow \infty$, with $\quad \mu=$ $\frac{\lambda_{c}^{\prime \prime}(s)}{2} \int_{-1}^{1} z^{2} K(z) d z, \sigma^{2}=$ $\tau^{2-b} \lambda_{c}(s) \zeta(b) \int_{-1}^{1} K^{2}(z) d z$ and $\zeta(b)=$ $\lim _{n \rightarrow \infty}\left(\sum_{k=1}^{\infty} \frac{1}{k^{b}} I(x+s+k \tau \in[0, n])\right)$.

The proofs of theorems above can be obtained through a rough analysis, (Maulidi, 2015). However, the basic theory to proof these theorems can be studied in(Dudley, 1989), (Hogg et al., 2005), and (Serfling, 1980).

The simulation is given by generating local intensity estimators at intervals $[0,20 \tau]$. It can be choosed other values of $\tau$, in this simulation we choose $\tau=5$. The method used is the Monte Carlo method. The purpose of this simulation is to see how convergence the variance and bias of the estimator.

The intensity function used in this simulation is

$$
\lambda(s)=\left[2 \exp \left(\sin \left(\frac{2 \pi s}{5}\right)\right)\right] s^{b}
$$

for $b=0.5, b=1$, and $b=2$. The chosen bandwidth is the optimal bandwidth that minimizes MSE, see [2], is as follows:

$h_{n}=$

$\left[\frac{\tau \lambda_{c}(s) \int_{-1}^{1} K^{2}(x) d x}{(1-b)\left(\lambda_{c}^{\prime \prime}(s) \int_{-1}^{1} x^{2} K(x) d x\right)^{2}}\right]^{\frac{1}{5}} n^{-(1,5) / 5}$, for $b=$

0.5 ,

$h_{n}=\left[\frac{\tau \lambda_{c}(s) \ln (n) \int_{-1}^{1} K^{2}(x) d x}{\left(\lambda_{c}^{\prime \prime}(s) \int_{-1}^{1} x^{2} K(x) d x\right)^{2}}\right]^{\frac{1}{5}} n^{-2 / 5}$,

for $b=1$,

$h_{n}=\left[\frac{\tau^{2-b} \lambda_{c}(s) \zeta(b) \int_{-1}^{1} K^{2}(x) d x}{\left(\lambda_{c}^{\prime \prime}(s) \int_{-1}^{1} x^{2} K(x) d x\right)^{2}}\right]^{\frac{1}{5}} n^{-2 / 5}$,

for $b=2$.

The kernel function that has been used in this simulation was a uniform kernel function $K(x)=\frac{1}{2},-1 \leq x \leq 1, x \in$ $\mathbb{R}$. The simulation used R program. The 
simulation algorithms are given as follows:

a. Generate the realization of periodic Poisson process at interval $[0, n]$ and period $\tau$.

b. Generate the estimator at a point $s$ with the bandwidth $h_{n}$ and uniform kernel.

c. Determine mean of the estimator, mean of the theory, variance of the estimator, variance of the theory, bias, and the difference between the variance of the estimator and variance of the theory.

\section{RESULTS AND DISCUSSION}

The results of the simulation for each case of the value $b$ with $\mathrm{s}=3$ are shown in Table 1, Table 2, and Table 3 as follows:

Table 1. The simulation results from the estimator generated and the theoretical results for $b=0.5$

\begin{tabular}{ccccccc}
\hline $\mathbf{n}$ & $\widehat{\lambda_{n, K}}$ theory & $\widehat{\lambda_{n, K} \text { simulation }}$ & $\widehat{\boldsymbol{\sigma}}^{2}$ theory & $\widehat{\boldsymbol{\sigma}}^{2}$ simulation & Bias & $\begin{array}{c}\widehat{\boldsymbol{\sigma}}^{2} \text { theory }-\widehat{\boldsymbol{\sigma}}^{2} \\
\text { simulation }\end{array}$ \\
100 & 1.9245 & 1.7431 & 0.1195 & 0.1796 & -0.1814 & 0.0600 \\
300 & 1.9245 & 1.8121 & 0.0496 & 0.0725 & -0.1124 & 0.0228 \\
500 & 1.9245 & 1.8570 & 0.0330 & 0.0473 & -0.0675 & 0.0143 \\
1000 & 1.9245 & 1.8833 & 0.0189 & 0.0269 & -0.0412 & 0.0101 \\
1500 & 1.9245 & 1.8951 & 0.0137 & 0.0207 & -0.0294 & 0.0070 \\
2000 & 1.9245 & 1.9048 & 0.0109 & 0.0174 & -0.0197 & 0.0174 \\
\hline
\end{tabular}

Table 2. The simulation results from the estimator generated and the theoretical results

\begin{tabular}{ccccccc}
\multicolumn{8}{c}{ for $b=1$} \\
$\mathbf{n}$ & $\widehat{\lambda_{n, \boldsymbol{K}}}$ theory & $\widehat{\lambda_{n, \boldsymbol{K}} \text { simulation }}$ & $\widehat{\widehat{\sigma}}^{\mathbf{2}}$ theory & $\widehat{\boldsymbol{\sigma}}^{2}$ simulation & Bias & $\begin{array}{c}\hat{\boldsymbol{\sigma}}^{2} \text { theory }-\widehat{\sigma}^{2} \\
\text { simulation }\end{array}$ \\
100 & 3.3333 & 1.7026 & 0.0069 & 0.0032 & -1.6307 & -0.0037 \\
300 & 3.3333 & 1.8458 & 0.0014 & 0.0008 & -1.4875 & -0.0006 \\
500 & 3.3333 & 1.8767 & 0.0007 & 0.0005 & -1.4566 & -0.0002 \\
1000 & 3.3333 & 1.9013 & 0.0002 & 0.0002 & -1.4321 & 0 \\
\hline
\end{tabular}

Table 3. The simulation results from the estimator generated and the theoretical results

\begin{tabular}{ccccccc}
\multicolumn{8}{c}{ for $b=2$} \\
$\mathbf{n}$ & $\widehat{\lambda_{\boldsymbol{n}, \boldsymbol{K}}}$ theory & $\widehat{\lambda_{\boldsymbol{n}, \boldsymbol{K}}}$ simulation & $\widehat{\boldsymbol{\sigma}}^{2}$ theory & $\widehat{\boldsymbol{\sigma}}^{2}$ simulation & Bias & $\begin{array}{c}\hat{\boldsymbol{\sigma}}^{2} \text { theory }-\hat{\boldsymbol{\sigma}}^{2} \\
\text { simulation }\end{array}$ \\
70 & 10.0000 & 5.4328 & 0.0011 & 0.0219 & -4.5672 & -0.0207 \\
80 & 10.0000 & 6.2890 & 0.0005 & 0.0177 & -3.7110 & -0.0172 \\
100 & 10.0000 & 6.6024 & 0.0004 & 0.0141 & -3.3976 & -0.0137 \\
120 & 10.0000 & 6.8237 & 0.0002 & 0.0103 & -3.1763 & -0.0101 \\
\hline
\end{tabular}

Table 1 shows the bias values and the difference between the variance of the theory and variance of the simulation. It can be seen that the bias in Table 1 converges to zero faster than the bias in Table 2 and Table 3 . The value of $b=$ 0.5 From this simulation, the larger values of $b$ requires a large interval of observation to determine the convergence of the estimator.

Unlike the bias, the variance values in Table 1 are slower to converge to zero when compared to Table 2 and Table 3. So that the greater value of $b$, than the smaller variance of the estimator. 
Desimal, 3 (3), 2020 - 275

Ikhsan Maulidi, Mahyus Ihsan, Vina Apriliani

$\lambda(s)$

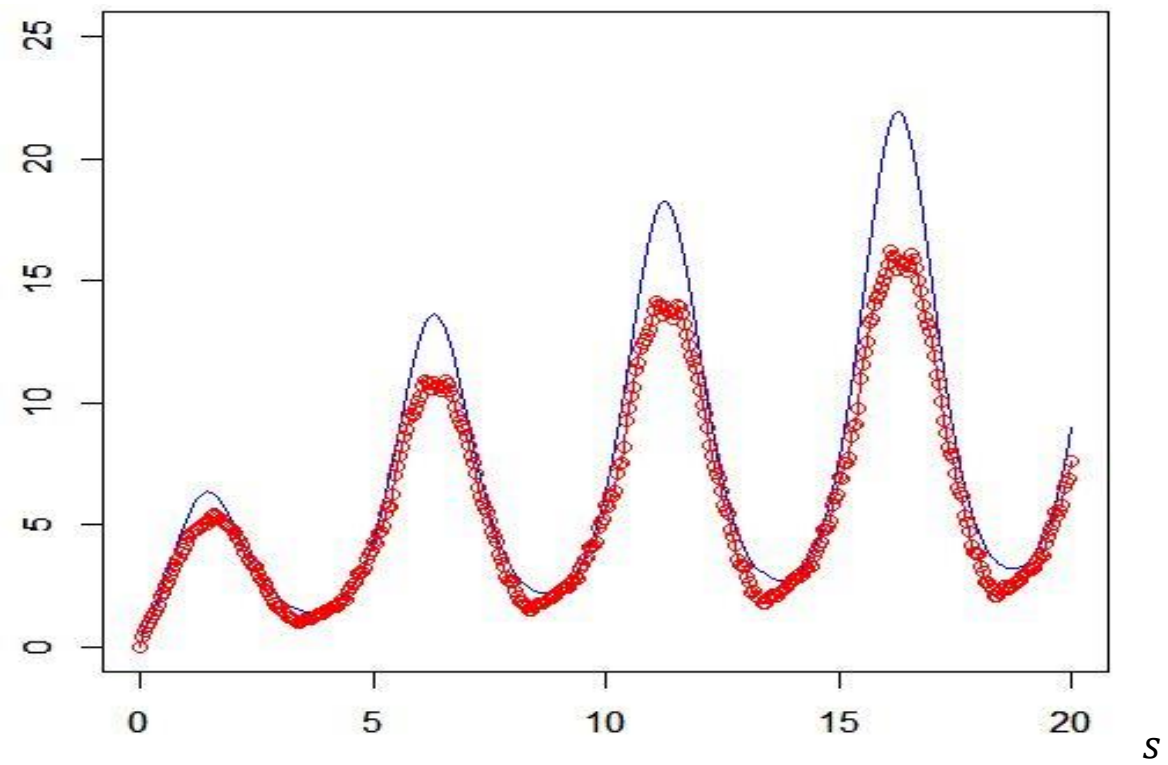

Figure 1.Graph of intensity function( the blue line) and its estimated value (the red line) with observation interval $[0,100]$ for $b=0.5$ using uniform kernel function

$\lambda(s)$

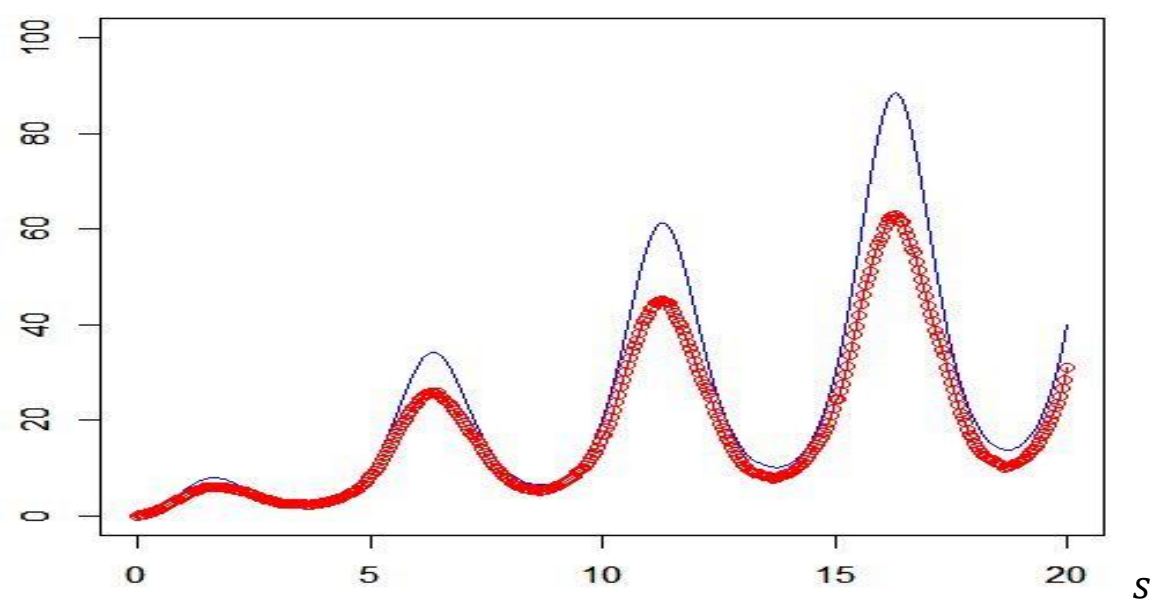

Figure 2.Graph of intensity function (the blue line) and its estimated value (the red line) with observation interval $[0,100]$ for $b=1$ using uniform kernel function 


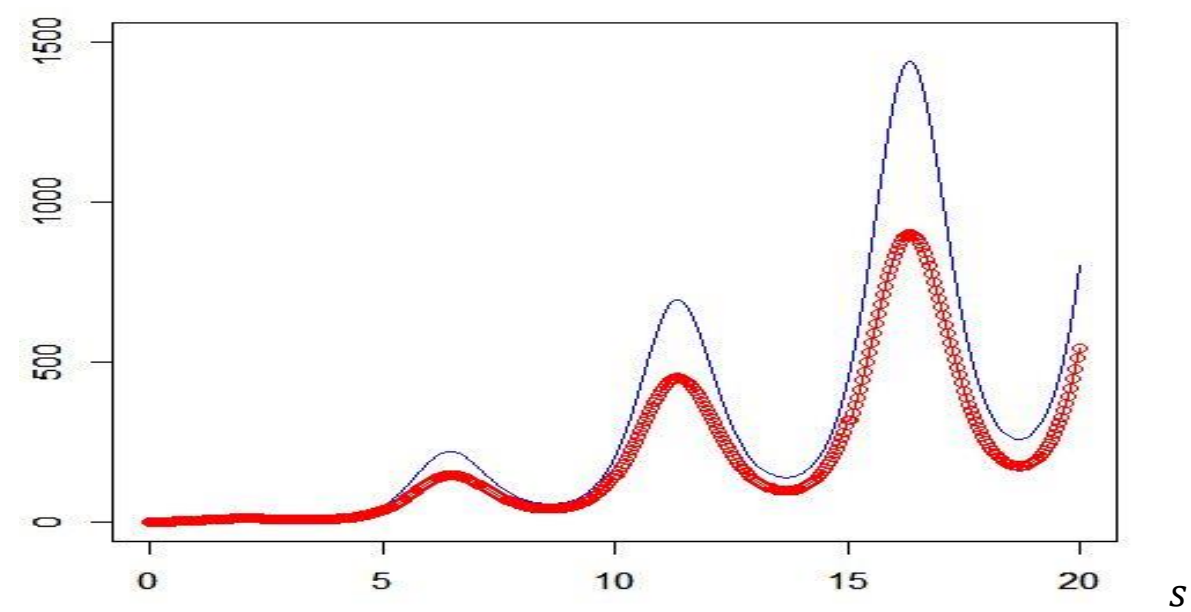

Figure 3. Graph of intensity function (the blue line) and its estimated value (the red line) with observation interval $[0,100]$ for $b=2$ using uniform kernel function

Figure 1, Figure 2, and Figure 3 show the alleged plot of the estimator and the actual intensity function. From the figure, it can be seen that the greater the value of $b$, then bias, which in this case represents the difference between the red points and the graph lines of intensity, is greater for the observation interval $[0,100]$.

\section{CONCLUSIONS AND SUGGESTIONS}

From the simulation that has been done to see the convergence rate of bias and variance of the estimator, the results show that the greater the value $b$, then the larger observation interval will be needed to achieve the convergence of estimators. This results should be proved theoretically by using estimator proposed and the Theorems related.

The suggestions for the next simulation are to provide a numerical simulation for each case of Theorem 1, Theorem 2, and Theorem 3 specifically. It could be done by taken the bandwidth function which satisfies the given conditions.

\section{REFERENCES}

Dudley, R. (1989). Real analysis and probability. Wardswort \& Brooks.

Erliana, W., Mangku, I. W., \& Sumarno, H. (2014). Estimating the intensity obtained as product of a periodic function with the power function trend of a nonhomogeneous poisson process. Far East Journal of Mathematical Sciences, 90(2), 163172.

Helmers, R., Wayan Mangku, I., \& Zitikis, R. (2003). Consistent estimation of the intensity function of a cyclic Poisson process. Journal of Multivariate Analysis, 84(1), 19-39. https://doi.org/10.1016/S0047259X(02)00008-8

Hogg, R., Craig, A., \& Kean, J. M. (2005). Introduction to mathematical statistics (6th ed.). Prentice Hall, Upper Saddle River.

Mahmud, T., Hasan, M., Chakraborty, A., \& Roy-chowdhury, A. K. (n.d.). Tahmida Mahmud University of California , Riverside. 2016 IEEE International Conference on Image Processing (ICIP), 3339-3343. https://doi.org/10.1109/ICIP.2016.7 532978

Mangku, I. W. (2011). Estimating the intensity obtained as the product of a periodic function with the linear tren of a non-homogeneous Poisson process. Far East Journal of Mathematical Science, 51, 141-150. Mangku, I. W., Budiarti, R., \& Taslim, C. 
Desimal, 3 (3), 2020 - 277

Ikhsan Maulidi, Mahyus Ihsan, Vina Apriliani

(2013). Estimating the intensity obtained as the product of a periodic function with the quadratic trend of a non-homogeneous poisson process. Far East Journal of Mathematical Science, 82(1), 33-44.

Maulidi, I. (2015). Kekonsistenan kuat dan sebaran asimtotik penduga intensitas berupa fungsi periodik kali tren fungsi pangkat proses poisson nonhomogen. IPB University.

Maulidi, I., Mangku, I. W., \& Sumarno, H. (2015). Strong consistency of kerneltype estimator for the intensity obtained as the product of a periodic function with the power function trend of non-homogeneous poisson process. British Journal of Applied Science and Technology, 9(4), 383387.

Serfling, R. (1980). Approximation theorems of mathematical statistics. J Wiley.

Sumiati, I., Rahmani, U., \& Supian, S. (2019). Application of the nonhomogeneous poisson process for counting earthquakes. 127(January 2016), 163-176.

Tse, K.-K. (2014). Some applications of the poisson process. Applied Mathematics, 05(19), 3011-3017. https://doi.org/10.4236/am.2014.5 19288 
Desimal, 3 (3), 2020 - 278

Ikhsan Maulidi, Mahyus Ihsan, Vina Apriliani 